\title{
Implications of functional anatomy on information processing in the deep cerebellar nuclei
}

\author{
Yuval Baumel', Gilad A. Jacobson' ${ }^{2}$ and Dana Cohen ${ }^{1,3 *}$ \\ 1 Gonda Interdisciplinary Brain Research Center, Bar Ilan University, Ramat Gan, Israel \\ 2 Friedrich Miescher Institute for Biomedical Research, Basel, Switzerland \\ 3 The Goodman Faculty of Life Sciences, Bar Ilan University, Ramat Gan, Israel
}

\section{Edited by:}

Egidio D'Angelo, University of Pavia,

Italy

\section{Reviewed by:}

Carlos D. Aizenman, Brown University, USA

Martha Bagnall, Salk Institute for

Biological Studies, USA

*Correspondence:

Dana Cohen, Gonda Interdisciplinary Brain Research Center, Bar llan

University, Ramat Gan 52900, Israel. e-mail:cohendan@biu.ac.il
The cerebellum has been implicated as a major player in producing temporal acuity. Theories of cerebellar timing typically emphasize the role of the cerebellar cortex while overlooking the role of the deep cerebellar nuclei (DCN) that provide the sole output of the cerebellum. Here we review anatomical and electrophysiological studies to shed light on the DCN's ability to support temporal pattern generation in the cerebellum. Specifically, we examine data on the structure of the DCN, the biophysical properties of DCN neurons and properties of the afferent systems to evaluate their contribution to DCN firing patterns. In addition, we manipulate one of the afferent structures, the inferior olive $(\mathrm{IO})$, using systemic harmaline injection to test for a network effect on activity of single DCN neurons in freely moving animals. Harmaline induces a rhythmic firing pattern of short bursts on a quiescent background at about $8 \mathrm{~Hz}$. Other neurons become quiescent for long periods (seconds to minutes). The observed patterns indicate that the major effect harmaline exerts on the DCN is carried indirectly by the inhibitory Purkinje cells (PCs) activated by the IO, rather than by direct olivary excitation. Moreover, we suggest that the DCN response profile is determined primarily by the number of concurrently active PCs, their firing rate and the level of synchrony occurring in their transitions between continuous firing and quiescence. We argue that DCN neurons faithfully transfer temporal patterns resulting from strong correlations in PCs state transitions, while largely ignoring the timing of simple spikes from individual PCs. Future research should aim at quantifying the contribution of PC state transitions to DCN activity, and the interplay between the different afferent systems that drive DCN activity.

Keywords: temporal patterns, rebound firing, short-term depression, harmaline, chronic recording, inferior olive, cerebellar nuclei

\section{ANATOMY OF THE DEEP CEREBELLAR NUCLEI TYPES OF NEURONS}

The deep cerebellar nuclei (DCN) consist of three nuclei: the fastigial (medial) nucleus, the interposed nucleus and the dentate (lateral) nucleus. Together they form the sole output of the cerebellum. The total number of DCN neurons has been estimated at about 50-100,000 (Heidary, 1972; Beitz and Chan-Palay, 1979; Green et al., 2006). The neurons can be divided into three main sub-categories: excitatory projection neurons constituting approximately $50-60 \%$ of the population, that project to a variety of extra-cerebellar targets, including the cerebral cortex via the thalamus (Batini et al., 1992; Schwarz and Schmitz, 1997; Holdefer et al., 2000; Teune et al., 2000; Middleton and Strick, 2001; Dum and Strick, 2003; Kelly and Strick, 2003); inhibitory projection neurons constituting approximately $30-35 \%$ of the population, that project exclusively to the inferior olive (Tolbert et al., 1976; De Zeeuw et al., 1989; Fredette and Mugnaini, 1991); and local inhibitory interneurons constituting less than $10 \%$ of DCN neurons (Chan-Palay, 1977; Czubayko et al., 2001; Aizenman et al., 2003). These three subgroups are intermixed and spatially distributed heterogeneously throughout the DCN (Beitz and Chan-Palay, 1979; Kumoi et al., 1988; Batini et al., 1992). Recently, glycinergic projection neurons unique to the fastigial nuclei have been described
(Bagnall et al., 2009). There are no reliable classifications of single cells into subgroups based solely on morphological features due to a considerable overlap in some of their features. For example, the soma size of the excitatory projection neurons ranges from 15 to 35 microns while that of the inhibitory ones ranges from 5 to 20 microns.

\section{THE INPUT TO THE DCN}

The DCN receive glutamatergic and GABAergic synaptic inputs, as well as a less-studied neuromodulatory input (see Gardette et al., 1987; Kitzman and Bishop, 1997; Saitow et al., 2009 for serotonergic innervations; and Jaarsma et al., 1997 for cholinergic innervations). The GABAergic input arises from PC axons. The glutamatergic input arises from the mossy fiber ( $\mathrm{mf}$ ) collaterals carrying information from the spinal cord, diverse areas in the cerebral cortex (via the dorsal pontine nuclei) and several brainstem areas, and the climbing fiber axon collaterals - the olivo-cerebellar (oc) fibers - which carry information from the IO (Kandel et al., 2000). Each type of input forms a different varicosity on DCN neurons, making it possible to sort synapses according to their origin. The largest portion of synaptic connections is formed by PCs [about $60-85 \%$ of synapses, depending on the species and method used for counting (Chan-Palay, 1973b; Mezey et al., 1977; Palkovits et al., 1977; 
De Zeeuw and Berrebi, 1996)]. The oc fibers constitute about 5\% of the DCN input (Chan-Palay, 1973b; Van der Want et al., 1989). The number of interneuron and $\mathrm{mf}$ synapses onto the projection neurons remains unclear (see e.g., Chan-Palay, 1973b; Wassef et al., 1986; De Zeeuw and Berrebi, 1995, 1996). The vast majority of axon terminals reaching the DCN form synaptic contacts on the glutamatergic projection neurons rather than on the GABAergic neurons ( $80 \%$ vs. $20 \%$ ). This ratio is maintained regardless of origin (De Zeeuw and Berrebi, 1996).

The convergence-divergence ratio of different afferent inputs to the DCN has been estimated only for the PC-DCN pathway. A single PC is estimated to innervate approximately 30-40 DCN neurons and in turn, each DCN neuron, receives projections from about 600-900 PC (Chan-Palay, 1973a; Mezey et al., 1977; Palkovits et al., 1977). It is estimated that most of PC axons innervate both glutamatergic and GABAergic cells (De Zeeuw and Berrebi, 1995, 1996; Teune et al., 1998). Generally it has been suggested that mf collaterals show considerable ramification and a bilateral projection pattern in the nuclei (Shinoda et al., 1992, 2000; Wu et al., 1999) whereas the oc distribution is more local, generating a closed loop composed of the IO - cerebellar cortex - DCN - IO (Sugihara et al., 1999, 2001; Shinoda et al., 2000; Sugihara and Shinoda, 2004; Pijpers et al., 2005).

The dominance of the PC input onto the DCN seems to suggest that it plays a major role in modulating DCN activity compared to the other inputs. In the next section, we review electrophysiological evidence regarding the relative contribution of the different inputs to DCN output.

\section{DCN ELECTROPHYSIOLOGY}

Neurons in the DCN receive a wide array of inputs, both excitatory and inhibitory. A central question is to which extent can the different kinds of input shape DCN output, and whether the $\mathrm{DCN}$ is sensitive to specific interactions between co-occurring inputs from these different sources. Attaining such an understanding would be a significant step toward underpinning the DCN computation.

Several studies have monitored neuronal activity from DCN neurons during sensory stimuli and motor activity. Neurons in the DCN tend to fire spontaneously at rates $>10 \mathrm{~Hz}$, and can modulate their firing rates in complex ways in response to either sensory or motor activation (e.g., Thach, 1968, 1975; Eccles et al., 1974b,c; Strick, 1983; Rowland and Jaeger, 2005, 2008). Neurons exhibit increases and decreases in firing rate, suggesting that both excitatory and inhibitory inputs to the DCN play a significant role in shaping its output. It is difficult, though, to conclude from such works which afferent structures and synaptic conductances generated these responses. Rowland and Jaeger (2008) have tried to correlate DCN firing profile with the activity in different afferent structures. Their results suggest that IO output can substantially affect DCN output. Nonetheless, their olivary signal was limited to local field potential, and the complexity of the olivo-nuclear connection, which has both direct and indirect paths, further complicates the interpretation of such results.

Further insight into DCN processing comes from testing the influence of temporary modification of the afferent systems on animal behavior. Injection of both GABA agonists and antagonists to the DCN abolishes conditioned eye blink responses (Aksenov et al., 2004), while blockade of fast glutamate receptors in the DCN have a minor effect on conditioned responses (Aksenov et al., 2005). These results suggest that the expression of eye blink conditioning depends on intact GABAergic, and not glutamatergic, pathways to the DCN.

DCN processing depends not only on the properties of DCN afferent systems but also on the biophysics of DCN neurons. One major observation is that the PC-DCN synapse exhibits a strong short-term depression (STD). STD is shown in Figure $\mathbf{1}$ in which a paired pulse protocol was used to stimulate the PC-DCN synapse. STD ranges from $30 \%$ for a $20-\mathrm{Hz}$ input to $90 \%$ for rates $>140 \mathrm{~Hz}$ (Telgkamp and Raman, 2002; Pedroarena and Schwarz, 2003). Thus, it seems that the massive PC-DCN projection is counteracted by a physiological mechanism, and may not be as dominant as indicated by anatomy. This may explain how neurons of
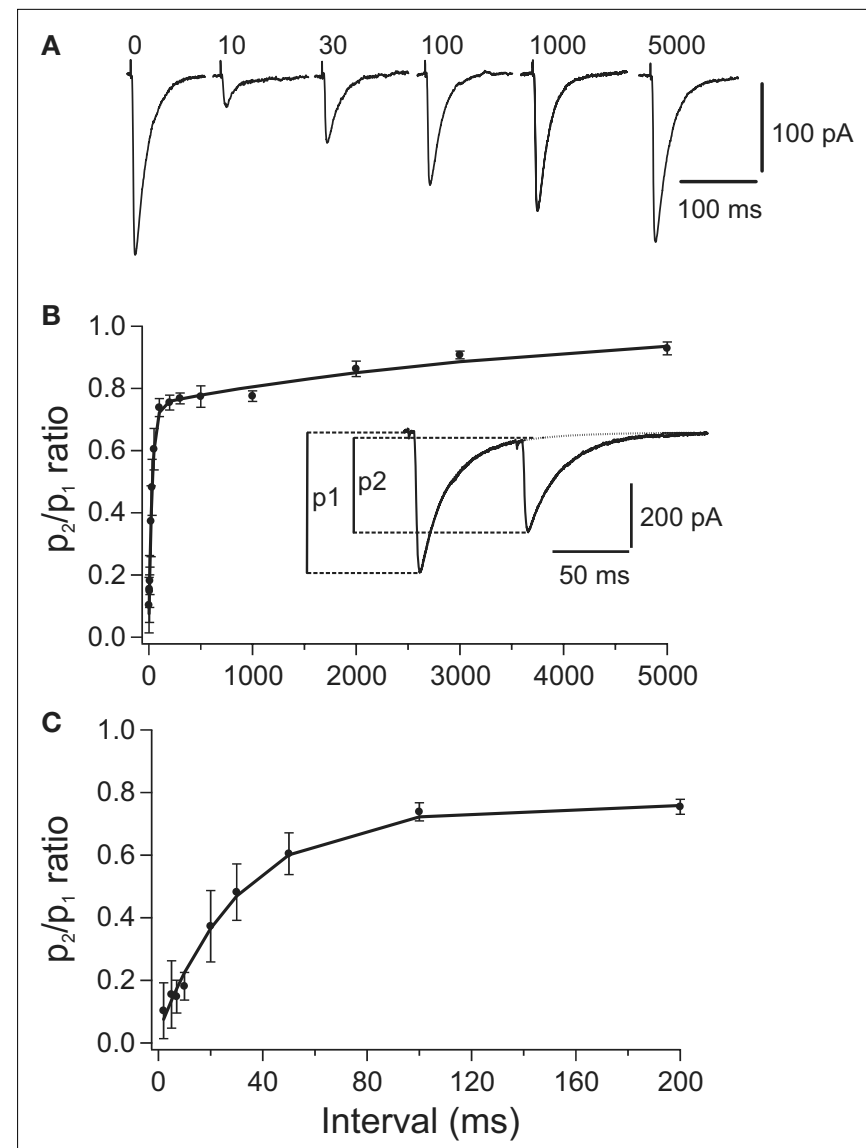

FIGURE 1 | Paired pulse depression (PPD) of compound PC-DCN IPSCs.

(A) Representative example of IPSCs evoked by paired stimulation. Each trace illustrates the average of 25 single IPSCs evoked in a DCN by pairs of stimuli with different intervals applied to PC axons. Number above each trace indicates the interpulse interval in milliseconds. Traces corresponding to 10 and $30 \mathrm{~ms}$ were obtained after subtracting the response to the $1 \mathrm{st}$ stimulus (labeled with "0"). (B,C) Time course of recovery from PPD shown at two different time scales. Average peak amplitude ratio (p2/p1) was plotted against the interpulse interval $(n=24)$. The curves represent a double exponential expression fitted to the data. The peak amplitude of IPSCs was measured from the baseline to the peak of the respective IPSCs. Taken with permission from Pedroarena and Schwarz (2003). 
the cerebellar nuclei maintain relatively high basal firing rates of 10-100 Hz (Thach, 1968; LeDoux et al., 1998; Rowland and Jaeger, 2005) despite the convergence of many PCs each firing at about $50 \mathrm{~Hz}$. Nonetheless, direct stimulation of the inhibitory pathway at a rate above the presumed PC firing rate can lead to a further decline in DCN firing rate, demonstrating that changes in PC activity still have the capacity to modulate DCN activity (Telgkamp and Raman, 2002). Overall, PC-DCN synapses seem to exhibit larger sensitivity to dynamic than to steady PC activation; and despite the significant depression at the PC-DCN synapse, PCs can provide effective inhibition and shape DCN firing patterns (Telgkamp and Raman, 2002; Pedroarena and Schwarz, 2003).

Synaptic depression at the PC-DCN synapse has several computational implications. First, basal levels of asynchronous inhibition may keep DCN neurons within their dynamic range thus allowing them to respond to increases, as well as decreases, in PC firing rate (Thach, 1968; Jahnsen, 1986; Gauck and Jaeger, 2000; Telgkamp and Raman, 2002; Pedroarena and Schwarz, 2003). Second, the significant decline in PC-DCN synaptic strength suggests that although PC axons can transfer spike doublets when firing a complex spike (CS) (Khaliq and Raman, 2005; Monsivais et al., 2005), the DCN response to the second spike in the doublet will be strongly depressed. This supports the idea that DCN neurons can respond only to the first spike in the CS and thus cannot distinguish it from the arrival of SSs. If this is indeed the case, STD enables the IO to generate a complex spatiotemporal activation of the cerebellar cortex including induction of LTD/LTP and controlling pause duration (Mathy et al., 2009) without maintaining the tagging of CS activity when transferred downstream to the DCN. The impact of the direct IO-DCN excitatory pathway on DCN has still to be thoroughly investigated. Last, an important implication of the fast depression is that DCN neurons should be highly sensitive to changes in the rate of PC input, as elaborated in the section "Discussion".

Another observation is that DCN neurons respond to a hyperpolarizing current with a post-hyperpolarization rebound response, which plays a major part in many cerebellar theories (Medina et al., 2000; Kistler and De Zeeuw, 2003; Wetmore et al., 2008); after hyperpolarization, the membrane rapidly depolarizes and is capable of producing spikes at membrane potentials lower than prior to hyperpolarization (Figure 2). The strength of the rebound response increases with the amplitude (Figures $2 \mathrm{~A}-\mathrm{C}$ ) and the duration (Figures 2D-F) of hyperpolarization (Jahnsen, 1986; Aizenman and Linden, 1999). This endows DCN neurons with the ability to respond to inhibitory inputs with a spiking output and also to respond differently to the same input depending on the history of synaptic input (Figures 2G,H). Thus, it is difficult to relate changes in firing rate to excitation/inhibition in a trivial way. The large temporal variability of about $50 \mathrm{~ms}$ in the onset of the rebound responses (Llinás and Muhlethaler, 1988) poses a problem for theories of cerebellar timing (Ivry and Keele, 1989; Mauk et al., 2000; Ivry and Spencer, 2004). This limitation may be
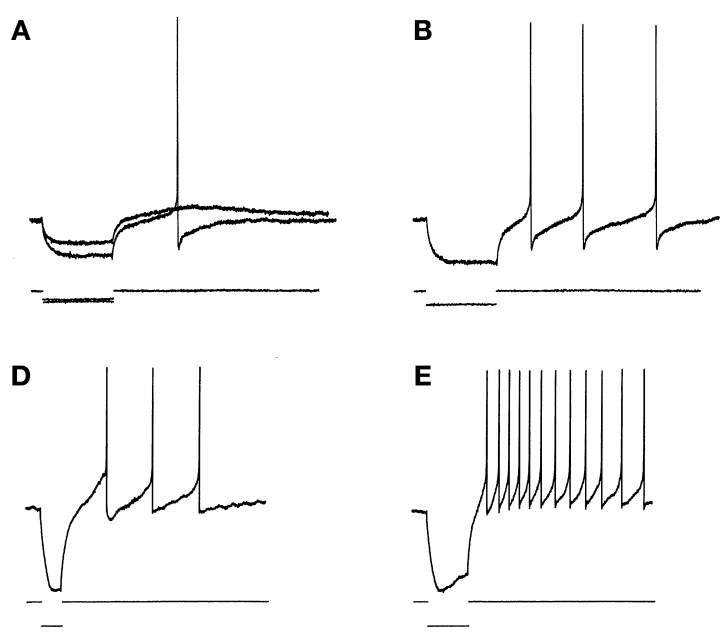
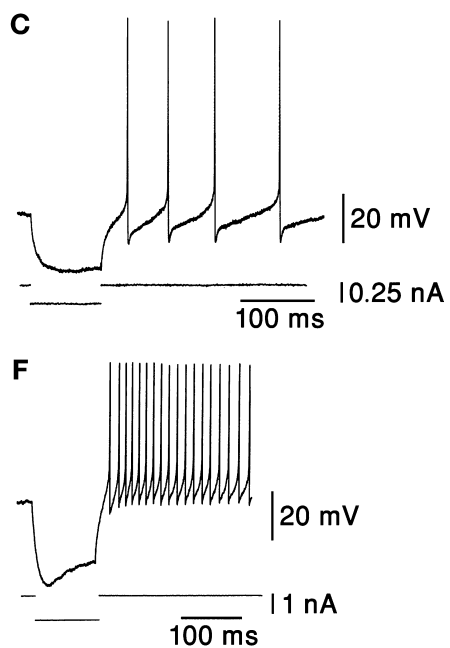

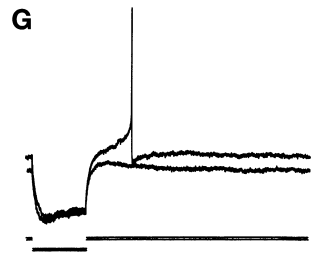

FIGURE 2 | Responses to hyperpolarizing current pulses. (A-C) Rebound responses were seen after hyperpolarizing current pulses. The smallest pulse produced only a subthreshold depolarization. As the stimulus increased in amplitude an increasing number of action potentials were generated. (D-F) The

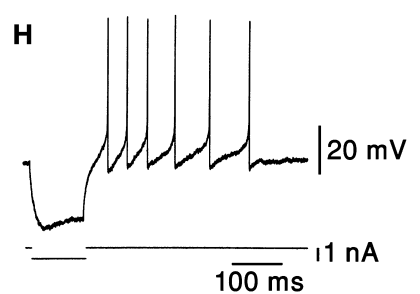

responses were dependent on the duration of preceding hyperpolarization. More spikes were seen after long hyperpolarizations. $(\mathbf{G}, \mathbf{H})$ The rebound response was dependent on the initial membrane potential. The response was larger when the membrane was depolarized. Taken with permission from Jahnsen (1986). 
resolved if active depolarization occurs in these neurons instead of a mere return to resting potential, or by a strong, purely excitatory input component (e.g., Gauck and Jaeger, 2003; Rowland and Jaeger, 2005).

Different neuronal populations in the DCN may possess different biophysical machinery and firing properties. Attempts to correlate morphological features of DCN neurons such as soma size and shape, and dendritic arborization with electrophysiological properties have until recently not been conclusive, mainly because of the overlap in morphological properties of different DCN cell types. Recently, an in vitro study that allowed separating the inhibitory projection neurons from the non-inhibitory ones demonstrated that a reliable classification of DCN cells into subgroups can be achieved using a combination of several electrophysiological properties (Uusisaari et al., 2007). Specifically, compared to the excitatory projection neurons, the inhibitory projection neurons have a lower spontaneous firing rate and they do not reach as high firing frequencies during depolarizing current injections. Furthermore, these cells exhibit a longer-lasting rebound depolarization and associated spiking after a transient hyperpolarization. Increasing the temperature in vitro enhanced the difference in rebound firing between the excitatory and inhibitory neuronal populations. This would imply that under normal body temperature these differences will be further enhanced. In such a case, two outcomes could be expected: (1) the rather short lasting rebound firing in the excitatory projection neurons will be capable of reporting neuronal signals with higher temporal acuity; and (2) the long rebound firing in the inhibitory projection neurons will be capable of providing stable tonic GABAergic input to control the strength of gap junction coupling in IO neurons for long time periods (Jacobson et al., 2008).

To test the functional convergence of DCN afferents we used systemic injections of harmaline (10-15 mg/kg) to manipulate both the direct oc inputs to the DCN and the indirect PC input converging on the DCN (Llinás and Volkind, 1973). Harmaline, which serves as an animal model for essential tremor, accentuates IO subthreshold oscillations, thus increasing cf firing rate to about $10 \mathrm{~Hz}$ (De Montigny and Lamarre, 1973; Llinás et al., 1974). This massive CS activity, which occurs synchronously in PCs, significantly decreases SS firing in many PCs (Lamarre et al., 1971; Llinás and Volkind, 1973; Llinás and Muhlethaler, 1988). We recorded DCN activity in freely moving rats $(n=3)$ using chronically implanted electrodes (for methods see the Figure 3 and Cohen and Nicolelis, 2004; Jacobson et al., 2009). Preliminary results show that harmaline induced a transition from stochastic firing to long periods of quiescence $(7 / 12$ cells) or to an ON/OFF firing pattern in which activity alternated between short bursts of action potentials and long quiescence periods (5/12 cells). This ON/OFF pattern repeated every 100-130 ms which is typical of IO interspike intervals following harmaline application (Llinás and Volkind, 1973; Jacobson et al., 2009). Neurons could alternate between the ON/OFF pattern and the prolonged quiescence. In 3/12 cells, a variety of firing patterns was observed, including an increase in firing rate and a stable firing rate. Examples of two cells recorded prior to and after harmaline injection are shown in Figure 3. Similar firing patterns have previously been described in an isolated brain stem-cerebellar preparation under different levels of hyperpolarization (Llinás and Muhlethaler, 1988). In that work, harmaline injection resulted in a repeating synaptic input pattern consisting of a short lasting (10-12 ms) excitatory post synaptic potential (EPSP) followed by a long lasting (80-100 ms) inhibitory post synaptic potential (IPSP). Occasionally DCN neurons exhibited only IPSPs, suggesting that the distribution of inhibitory potentials over the cerebellar nucleus is broader than that of the excitatory oc potentials (Llinás and Muhlethaler, 1988). This in turn suggests that the direct effect of IO on DCN via oc collaterals is probably more restricted than the IO broad effect via PCs. The extent and conditions under which direct oc input can induce an excitatory response in DCN neurons remain to be determined.

\section{DISCUSSION}

Information processing depends on structural anatomy, intrinsic properties of the neuronal elements and network dynamics. In this review, we summarize the major points relating to these aspects of DCN processing and further provide initial results relating to DCN network performance by examining harmaline-induced changes in DCN activity in freely moving animals.

At first glance, it seems likely that as a result of the increase in IO firing induced by harmaline (Lamarre et al., 1971; Llinás and Volkind, 1973; Llinás and Muhlethaler, 1988) activity in the DCN would be enhanced significantly via two different pathways: (1) excessive activation of oc collaterals is expected to excite DCN neurons and (2) cf activation of PC, which decreases significantly the firing of SS (Lamarre et al., 1971; Llinás and Volkind, 1973; Llinás and Muhlethaler, 1988), is expected to decrease PC firing rate and thus indirectly disinhibit the DCN. Our results, however, suggest that the effect of the IO on DCN firing is not as trivial. Instead of increasing their firing rate after harmaline application, $75 \%$ of the recorded the DCN neurons became quiescent or exhibited rhythmic ON/OFF firing patterns entrained to IO firing. Two neural mechanisms that are not mutually exclusive can induce the $\mathrm{ON}$ response: (1) direct activation of synchronous oc collaterals, and (2) rebound firing in the DCN. Evidence for a role for rebound firing in shaping the ON response comes from Llinás and Muhlethaler (1988), showing that the duration of the $\mathrm{ON}$ response is significantly longer than that of the IO mediated EPSP and that strong phasic firing occurs following hyperpolarizing current injection. On the other hand, rebound firing has been questioned in vivo (Alvina et al., 2008) and there exists evidence for direct IO-DCN excitation (Eccles et al., 1974a,b; Rowland and Jaeger, 2005). The physiological conditions under which rebound firing manifests itself and the degree to which direct IO excitation can drive DCN neurons remain to be elucidated.

The paradoxical silencing of DCN neurons by harmaline can be attributed to several mechanisms: (1) inhibition exerted by cf-activated PCs; (2) effective recruitment of local DCN interneurons; or (3) depolarization block resulting from the reduction in PC firing. There is currently no clear evidence for any of these options. Local interneurons constitute a small group of DCN neurons, and their role so far remains unknown. Depolarization block has been reported only incidentally (Pugh and Raman, 2009), and the conditions under which it would manifest itself are yet to be determined. As for the inhibition exerted by cf-activated PCs, it is possible that the infrequent complex spikes produced by PCs on a silent background allow the PC-DCN inhibitory synapses to 


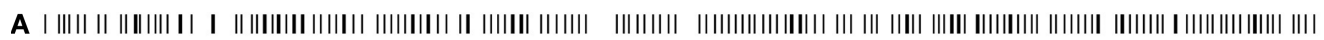

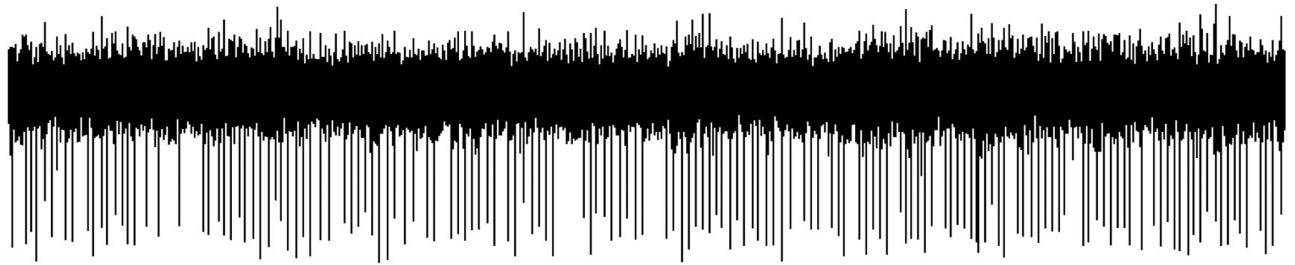

$$
1 \text { sec }
$$

B

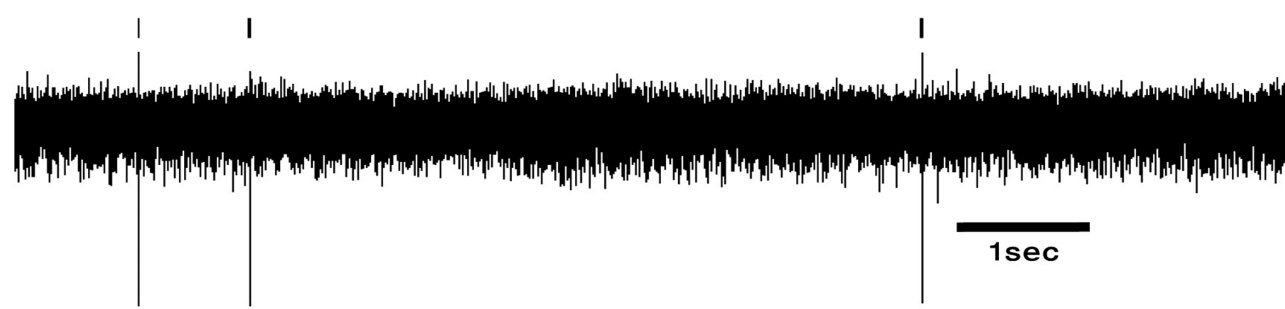

C

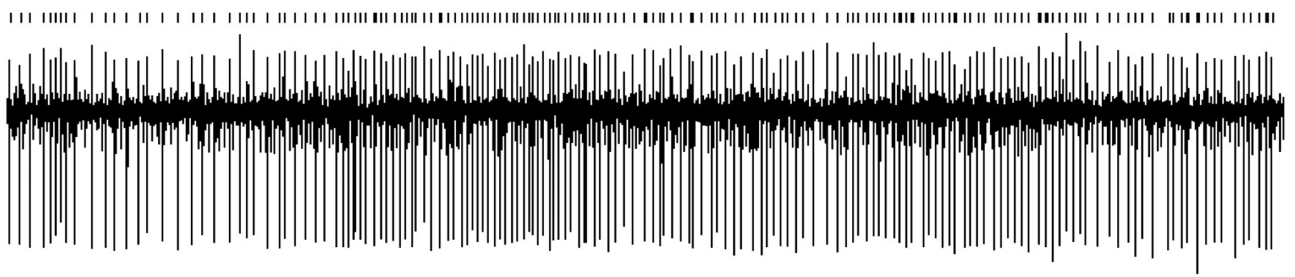

$250 \mathrm{~ms}$

D
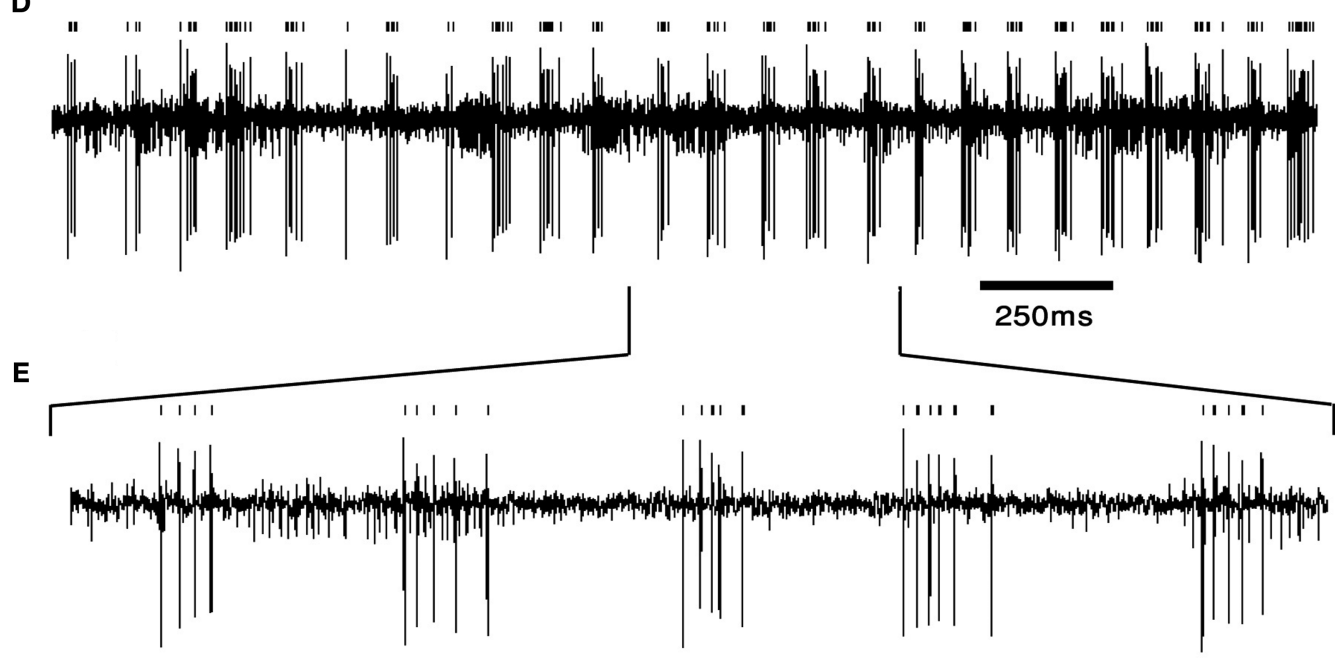

$50 \mathrm{~ms}$

FIGURE 3 | DCN neurons exhibit typical firing patterns following harmaline injection. $(\mathbf{A}, \mathbf{B})$ An example of a tonically firing neuron $(\mathbf{A})$ that became almost completely quiescent after harmaline injection (B). (C,D) An example of a tonically firing neuron (C) that exhibited an ON/OFF firing pattern after harmaline injection (D). (E) Time expansion of the marked section in (D) showing the typical cycle of the ON/OFF pattern. Small bars above the trace mark the sorted spikes. The surgical procedure has been described in detail (Cohen and Nicolelis, 2004; Jacobson et al., 2009). In brief, adult Long Evans male rats weighing 350$500 \mathrm{~g}$ (Harlan, Indianapolis, IN, USA) were initially sedated by $5 \%$ isoflurane and then injected i.m. with ketamine $\mathrm{HCl}$ and xylazine $\mathrm{HCl}(100$ and $10 \mathrm{mg} / \mathrm{kg}$, respectively). Supplementary injections of ketamine and xylazine were given as required. The skull surface was exposed and a craniotomy, slightly larger than the electrode array, was performed above the medial and interposed nuclei. Center of implant was at $-11.3 \mathrm{~mm}$ posterior to bregma, $1.5 \mathrm{~mm}$ lateral to the midline (coordinates taken from Paxinos and Watson, 1998). Arrays of 16 electrodes were lowered $4 \mathrm{~mm}$ from the surface of the brain and fixed using dental cement. Rats were allowed at least 2 weeks of recovery prior to recording. Electrode location was verified using histology. 
recover and exert a stronger inhibitory effect. This recovery by itself may not be enough, as in vitro studies have shown that inhibition grows with PC rate, despite STD. However, if complemented by harmaline induced recruitment of PCs that are normally silent, PC-DCN release-from-depression by CS activity may turn out to be highly effective.

What generates the heterogeneity in DCN responses to harmaline injection? The quiescent and rhythmic DCN neurons under harmaline (see Figure 3) may differ in the degree of synchronicity between the PCs that drive them, or simply in the number of rhythmic PCs impinging upon them at a given time. If the CS signals reach the DCN almost simultaneously, inhibition strength may wear off before the next CS, thus allowing for a rebound response. If on the other hand, CS signals arrive asynchronously throughout IO cycle, the inhibitory time-course will be smoothed, preventing rebound firing throughout the oscillatory cycle. Both zero lag (synchronous) and non-zero lags (asynchronous) IO activity correlations have been reported in vivo (Sugihara et al., 1993, 1995; Lang, 2001, 2002; Jacobson et al., 2009), providing a substrate for these two firing patterns. Alternatively, DCN neurons that receive less inhibition may be able to respond to the direct IO-DCN excitatory input, while strongly inhibited neurons may not.

What interplay between DCN afferents and biophysical properties would best account for the firing patterns observed in the absence of harmaline? The firing patterns observed in DCN in vivo suggest that the properties of the different afferent inputs and the biophysics of DCN neurons interact in complex ways to form the DCN output. Evidence exists for the involvement of all afferent structures in shaping DCN output (Thach, 1968; Eccles et al., 1974a,b; Strick, 1983; Rowland and Jaeger, 2005, 2008; Ohyama et al., 2006). The contribution of PCs to DCN firing is particularly interesting because PCs participate in the feedback loops involving both excitatory cerebellar afferents. Our results may indicate that normal cerebellar activation involves strong PC activation that in turn can bidirectionally modulate DCN firing.

To efficiently activate phasic firing in DCN neurons, converging PCs must start at a high firing rate to evoke hyperpolarization and cease firing synchronously to instantly remove inhibition and evoke a rebound response. Interestingly, increased synchrony has been reported in PCs following sensory stimulation (Schultz et al., 2009). Although the precise numbers are hard to pin down, we suggest that the firing patterns in DCN are controlled primarily by the number of concurrently active PCs, their firing rate and level of synchrony in the transition from continuous firing to quiescence.

Two plausible mechanisms may underlie synchronized intermissions lasting tens of milliseconds to seconds in PCs: (1) transition between states in bistable PCs imposed by synchronized IO

\section{REFERENCES}

Aizenman, C. D., Huang, E. J., and Linden, D. J. (2003). Morphological correlates of intrinsic electrical excitability in neurons of the deep cerebellar nuclei. J. Neurophysiol. 89, 1738-1747.

Aizenman, C. D., and Linden, D. J. (1999). Regulation of the rebound depolarization and spontaneous firing patterns of deep nuclear neurons in slices of rat cerebellum. J. Neurophysiol. 82, 1697-1709.

Aksenov, D., Serdyukova, N., Irwin, K., and Bracha, V. (2004). GABA neurotransmission in the cerebellar interposed nuclei: involvement in classically conditioned eyeblinks and neuronal activity. J. Neurophysiol. 91, 719-727.

Aksenov, D. P., Serdyukova, N. A., Bloedel, J. R., and Bracha, V. (2005). Glutamate

activity (Loewenstein et al., 2005; Jacobson et al., 2008; Yartsev et al., 2009), and (2) generation of pauses in SS firing in several PCs simultaneously by synchronized activation of molecular layer interneurons forming an efficient inhibitory network via gap junction (Mann-Metzer and Yarom, 1999; Mittmann et al., 2005). Analysis of SS activity in anesthetized animals reveals high synchrony prior to the occurrence of a pause (Shin and De Schutter, 2006; De Schutter and Steuber, 2009). This short lasting synchrony indicates that many PCs undergo an intermission in firing at the same time, thus generating an accurate timing signal marking the imminent removal of the sustained PC inhibition from DCN neurons.

The two mechanisms proposed for DCN disinhibition - SS pauses and PC bistability - differ in their duration. Short, pauseinduced intermissions may disinhibit DCN neurons just enough to generate a short burst of activity, while longer, state-transition intermissions may enable prolonged DCN firing which in turn can decouple IO neurons and reselect functional subgroups within it (Llinás, 1974; Llinás et al., 1974; De Zeeuw et al., 1989, 1993, 1997; Llinás and Sasaki, 1989; Lang et al., 1996; Placantonakis et al., 2006).

\section{FUTURE DIRECTIONS}

Despite significant advances in the last decades, the current understanding of DCN function is partial, and lags behind the research of the cerebellar cortex. Considering the crucial position of the DCN as the output station of the entire cerebellar circuit, improving our understanding of DCN function should be a central goal of cerebellar research.

Filling in the gaps in anatomical and physiological knowledge of the DCN is an important task but we believe that good hypotheses are necessary in order to interpret the data and design experiments that easily distinguish between different hypotheses.

We suggest two experiments that directly arise from our hypothesis that PC state transitions determine DCN output. The first is to monitor the activity of a DCN neuron simultaneously with its afferent PCs, and test the relationship between state transitions and DCN firing. The other is to study the effect of IO decoupling on DCN output. We expect IO decoupling to desynchronize state transitions in neighboring PCs leading to a smoothing of firing patterns in the DCN. We believe that in the upcoming decade, the DCN may stop stuttering and start telling a story.

\section{ACKNOWLEDGMENTS}

Dana Cohen was supported by grant SENSOPAC (FP6-IST028056) from the European Commission. Gilad A. Jacobson is supported by an EMBO long-term fellowship.

neurotransmission in the cerebellar interposed nuclei: involvement in classically conditioned eyeblinks and neuronal activity. J. Neurophysiol. 93, 44-52.

Alvina, K., Walter, J. T., Kohn, A., EllisDavies, G., and Khodakhah, K. (2008). Questioning the role of rebound firing in the cerebellum. Nat. Neurosci. 11, 1256-1258.
Bagnall, M. W., Zingg, B., Sakatos, A., Moghadam, S. H., Zeilhofer, H. U., and du Lac, S. (2009). Glycinergic projection neurons of the cerebellum. J. Neurosci. 29, 10104-10110.

Batini, C., Compoint, C., BuisseretDelmas, C., Daniel, H., and Guegan, M. (1992). Cerebellar nuclei and the nucleocortical projections in the rat: retrograde tracing coupled to GABA 
and glutamate immunohistochemistry. J. Comp. Neurol. 315, 74-84.

Beitz, A. J., and Chan-Palay, V. (1979). The medial cerebellar nucleus in the rat: nuclear volume, cell number, density and orientation. Neuroscience 4, 31-45.

Chan-Palay, V. (1973a). Afferent axons and their relations with neurons in the nucleus lateralis of the cerebellum: a light microscopic study.Z. Anat. Entwicklungsgesch. 142, 1-21.

Chan-Palay, V. (1973b). On the identification of the afferent axon terminals in the nucleus lateralis of the cerebellum. An electron microscope study.Z. Anat. Entwicklungsgesch. 142, 149-186.

Chan-Palay, V. (1977). Cerebellar Dentate Nucleus; Organization, Cytology and Transmitters. Berlin, Springer-Verlag.

Cohen, D., and Nicolelis, M. A. (2004), Reduction of single-neuron firing uncertainty by cortical ensembles during motor skill learning. J. Neurosci. 24, 3574-3582.

Czubayko, U., Sultan, F., Thier, P., and Schwarz, C. (2001). Two types of neurons in the rat cerebellar nuclei as distinguished by membrane potentials and intracellular fillings. J. Neurophysiol. 85 , 2017-2029.

De Montigny, C., and Lamarre, Y. (1973). Rhythmic activity induced by harmaline in the olivo-cerebello-bulbar system of the cat. Brain Res. 53, 81-95.

De Schutter, E., and Steuber, V. (2009). Patterns and pauses in Purkinje cell simple spike trains: experiments, modeling and theory. Neuroscience. $162,816-826$.

De Zeeuw, C. I., and Berrebi, A. S. (1995). Postsynaptic targets of Purkinje cell terminals in the cerebellar and vestibular nuclei of the rat. Eur. J. Neurosci. 7, 2322-2333.

De Zeeuw, C. I., and Berrebi, A. S. (1996). Individual Purkinje cell axons terminate on both inhibitory and excitatory neurons in the cerebellar and vestibular nuclei. Ann. N. Y. Acad. Sci. 781, 607-610.

De Zeeuw, C. I., Holstege, J. C., Ruigrok, T. J., and Voogd, J. (1989). Ultrastructural study of the GABAergic, cerebellar, and mesodiencephalic innervation of the cat medial accessory olive: anterograde tracing combined with immunocytochemistry.J. Comp. Neurol. 284, 12-35.

De Zeeuw, C. I., Van Alphen, A. M., Hawkins, R. K., and Ruigrok, T. J. H. (1997). Climbing fibre collaterals contact neurons in the cerebellar nuclei that provide a GABAergic feedback to the inferior olive. Neuroscience 80 , 981-986.

De Zeeuw, C.I., Wentzel, P., and Mugnaini, E. (1993). Fine structure of the dor- sal cap of the inferior olive and its GABAergic and non-GABAergic input from the nucleus prepositus hypoglossi in rat and rabbit. J. Comp. Neurol. 327, 63-82.

Dum, R. P., and Strick, P. L. (2003). An unfolded map of the cerebellar dentate nucleus and its projections to the cerebral cortex. J. Neurophysiol. 89, 634-639.

Eccles, J.C., Rantucci, T., Sabah, N.H., and Taborikova, H. (1974a). Somatotopic studies on cerebellar fastigial cells. Exp. Brain Res. 19, 100-118.

Eccles, J.C., Sabah, N. H., and Taborikova, H. (1974b). Excitatory and inhibitory responses of neurones of the cerebellar fastigial nucleus. Exp. Brain Res. 19, 61-77.

Eccles, J.C., Sabah, N. H., and Taborikova, H. (1974c). The pathways responsible for excitation and inhibition of fastigial neurones. Exp. Brain Res. 19, 78-99.

Fredette, B. J., and Mugnaini, E. (1991). The GABAergic cerebello-olivary projection in the rat. Anat. Embryol. (Berl.) 184, 225-243.

Gardette, R., Krupa, M., and Crepel, F. (1987).Differential effects of serotonin on the spontaneous discharge and on the excitatory amino acid-induced responses of deep cerebellar nucleineurons in rat cerebellar slices. Neuroscience 23, 491-500.

Gauck, V., and Jaeger, D. (2000). The control of rate and timing of spikes in the deep cerebellar nuclei by inhibition. $J$. Neurosci. 20, 3006-3016.

Gauck, V., and Jaeger, D. (2003). The contribution of NMDA and AMPA conductances to the control of spiking in neurons of the deep cerebellar nuclei. J. Neurosci. 23, 8109-8118.

Green, J. T., Arenos, J. D., and Dillon, C. J. (2006). The effects of moderate neonatal ethanol exposure on eyeblink conditioning and deep cerebellar nuclei neuron numbers in the rat. Alcohol 39, 135-150.

Heidary, H. (1972). Neuron populations in the cerebellum of the cat. Experientia 28, 313-314.

Holdefer, R. N., Miller, L. E., Chen, L. L., and Houk, J. C. (2000). Functional connectivity between cerebellum and primary motor cortex in the awake monkey. J. Neurophysiol. 84, 585-590.

Ivry, R., and Keele, S. (1989). Timing functions of the cerebellum. J. Cogn. Neurosci. 1, 136-152.

Ivry, R. B., and Spencer, R. M. C. (2004). The neural representation of time. Curr. Opin. Neurobiol. 14, 225-232.

Jaarsma, D., Ruigrok, T.J.,Caffe, R., Cozzari, C., Levey, A.I., Mugnaini, E., and Voogd, J. (1997). Cholinergic innervation and receptors in the cerebellum. Prog. Brain Res. 114, 67-96.
Jacobson, G. A., Lev, I., Yarom, Y., and Cohen, D. (2009). Invariant phase structure of olivo-cerebellar oscillations and its putative role in temporal pattern generation. Proc. Natl. Acad. Sci. U.S.A. 106, 3579-3584.

Jacobson, G. A., Rokni, D., and Yarom, Y. (2008). A model of the olivo-cerebellar system as a temporal pattern generator. Trends Neurosci. 31, 617-625.

Jahnsen, H. (1986). Electrophysiological characteristics of neurones in the guinea-pig deep cerebellar nuclei in vitro. J. Physiol. 372, 129-147.

Kandel,E. R., Schwartz,J.H., and Jessell, T. M. (eds). (2000). Principles of Neural Science. New York, McGraw-Hill.

Kelly, R. M., and Strick, P. L. (2003). Cerebellar loops with motor cortex and prefrontal cortex of a nonhuman primate. J. Neurosci. 23, 8432-8444.

Khaliq, Z. M., and Raman, I. M. (2005) Axonal propagation of simple and complex spikes in cerebellar Purkinje neurons. J. Neurosci. 25, 454-463.

Kistler,W.M., and De Zeeuw, C. I. (2003). Time windows and reverberating loops: a reverse-engineering approach to cerebellar function. Cerebellum 2, 44-54.

Kitzman, P. H., and Bishop, G. A. (1997) The physiological effects of serotonin on spontaneous and amino acid-induced activation of cerebellar nuclear cells: an in vivo study in the cat. Prog. Brain Res. 114, 209-223.

Kumoi, K., Saito, N., Kuno, T., and Tanaka, C. (1988). Immunohistochemical localization of gamma-aminobutyric acid- and aspartate-containing neurons in the rat deep cerebellar nuclei. Brain Res. 439, 302-310.

Lamarre, Y., de Montigny, C., Dumont, M., and Weiss, M. (1971). Harmalineinduced rhythmic activity of cerebellar and lower brain stem neurons. Brain Res. 32, 246-250.

Lang, E. J. (2001). Organization of olivocerebellar activity in the absence of excitatory glutamatergic input. $J$. Neurosci. 21, 1663-1675.

Lang, E. J. (2002). GABAergic and glutamatergic modulation of spontaneous and motor-cortex-evoked complex spike activity. J. Neurophysiol. 87, 1993-2008.

Lang, E. J., Sugihara, I., and Llinás, R. (1996). GABAergic modulation of complex spike activity by the cerebellar nucleoolivary pathway in rat. $J$. Neurophysiol. 76, 255-275.

LeDoux, M. S., Hurst, D. C., and Lorden, J. F. (1998). Single-unit activity of cerebellar nuclear cells in the awake genetically dystonic rat. Neuroscience 86, 533-545.

Llinás, R. (1974). Eighteenth Bowditch lecture. Motor aspects of cerebellar control. Physiologist 17, 19-46.
Llinás, R., Baker, R., and Sotelo, C. (1974). Electrotonic coupling between neurons in cat inferior olive.J Neurophysiol 37, 560-571.

Llinás, R., and Muhlethaler, M. (1988). Electrophysiology of guinea-pig cerebellar nuclear cells in the in vitro brain stem-cerebellar preparation. J. Physiol. 404, 241-258.

Llinás, R., and Sasaki, K. (1989). The functional organization of the olivocerebellar system as examined by multiple Purkinje cell recordings. Eur. J. Neurosci. 1, 587-602.

Llinás, R., and Volkind, R. A. (1973). The olivo-cerebellar system: functional properties as revealed by harmalineinduced tremor. Exp. Brain Res. 18, 69-87.

Loewenstein,Y.,Mahon, S., Chadderton, P., Kitamura, K., Sompolinsky, H., Yarom, Y., and Hausser, M. (2005). Bistability of cerebellar Purkinje cells modulated by sensory stimulation. Nat. Neurosci. 8, 202-211.

Mann-Metzer, P., and Yarom, Y. (1999). Electrotonic coupling interacts with intrinsic properties to generate synchronized activity in cerebellar networks of inhibitory interneurons.J. Neurosci. 19, 3298-3306.

Mathy, A., Ho, S. S., Davie, J. T., Duguid, I. C., Clark, B. A., and Hausser, M. (2009). Encoding of oscillations by axonal bursts in inferior olive neurons. Neuron 62,388-399.

Mauk, M. D., Medina, J. F., Nores, W. L., and Ohyama, T. (2000). Cerebellar function: coordination, learning or timing?' Curr. Biol. 10, R522-R525.

Medina, J.F., Nores, W. L., Ohyama, T., and Mauk, M. D. (2000). Mechanisms of cerebellar learning suggested by eyelid conditioning. Curr. Opin. Neurobiol. 10, 717-724.

Mezey, E., Palkovits, M., Hamori, J., and Szentagothai, J. (1977). Quantitative cytology and electron microscopy of the cerebellar nuclei in the cat. Verh. Anat. Ges. 71, 171-176.

Middleton, F. A., and Strick, P. L. (2001). Cerebellar projections to the prefrontal cortex of the primate. J. Neurosci. 21, 700-712.

Mittmann, W., Koch, U., and Hausser, M. (2005). Feed-forward inhibition shapes the spike output of cerebellar Purkinje cells. J. Physiol. 563, 369-378.

Monsivais, P., Clark, B. A., Roth, A., and Hausser, M. (2005). Determinants of action potential propagation in cerebellar Purkinje cell axons. J. Neurosci. $25,464-472$.

Ohyama, T., Nores, W. L., Medina, J. F., Riusech, F.A., and Mauk, M.D. (2006). Learning-induced plasticity in deep cerebellar nucleus. J. Neurosci. 26, 12656-12663. 
Palkovits, M., Mezey, E., Hamori, J., and Szentagothai, J. (1977). Quantitative histological analysis of the cerebellar nuclei in the cat. I. Numerical data on cells and on synapses. Exp. Brain Res. 28, 189-209.

Paxinos, G., and Watson, C. (1998). The rat brain in stereotaxic coordinates. San Diego: Academic press.

Pedroarena, C. M., and Schwarz, C. (2003). Efficacy and short-term plasticity at GABAergic synapses between Purkinje and cerebellar nuclei neurons. J. Neurophysiol. 89, 704-715.

Pijpers, A., Voogd, J., and Ruigrok, T. J. (2005). Topography of olivo-corticonuclear modules in the intermediate cerebellum of the rat. J. Comp. Neurol. 492, 193-213.

Placantonakis, D. G., Bukovsky, A. A., Aicher, S. A., Kiem, H.-P., and Welsh, J. P. (2006). Continuous electrical oscillations emerge from a coupled network: a study of the inferior olive using lentiviral knockdown of connexin36. J. Neurosci. 26, 5008-5016.

Pugh, J. R., and Raman, I. M. (2009). Nothing can be coincidence: synaptic inhibition and plasticity in the cerebellar nuclei. Trends Neurosci. 32, 170-177.

Rowland, N. C., and Jaeger, D. (2005). Coding of tactile response properties in the rat deep cerebellar nuclei. $J$. Neurophysiol. 94, 1236-1251.

Rowland, N. C., and Jaeger, D. (2008). Responses to tactile stimulation in deep cerebellar nucleus neurons result from recurrent activation in multiple pathways. J. Neurophysiol. 99, 704-717.

Saitow, F., Murano, M., and Suzuki, H. (2009). Modulatory effects of serotonin on GABAergic synaptic transmission and membrane properties in the deep cerebellar nuclei. J. Neurophysiol. 101, 1361-1374.

Schultz, S. R., Kitamura, K., PostUiterweer, A., Krupic, J., and Hausser, M. (2009). Spatial pattern coding of sensory information by climbing fiber- evoked calcium signals in networks of neighboring cerebellar Purkinje cells. J. Neurosci. 29, 8005-8015.

Schwarz, C., and Schmitz, Y. (1997). Projection from the cerebellar lateral nucleus to precerebellar nuclei in the mossy fiber pathway is glutamatergic: a study combining anterograde tracing with immunogold labeling in the rat.J. Comp. Neurol. 381, 320-334.

Shin, S.-L., and De Schutter, E. (2006). Dynamic synchronization of Purkinje cell simple spikes. J. Neurophysiol. 96, 3485-3491.

Shinoda, Y., Sugihara, I., Wu, H. S., and Sugiuchi, Y. (2000). The entire trajectory of single climbing and mossy fibers in the cerebellar nuclei and cortex. Prog. Brain Res. 124, 173-186.

Shinoda, Y., Sugiuchi, Y., Futami, T., and Izawa, R. (1992). Axon collaterals of mossy fibers from the pontine nucleus in the cerebellar dentate nucleus. $J$. Neurophysiol. 67, 547-560.

Strick, P.L.(1983). The influence of motor preparation on the response of cerebellar neurons to limb displacements. J. Neurosci. 3, 2007-2020.

Sugihara, I., Lang, E. J., and Llinás, R. (1993). Uniform olivocerebellar conduction time underlies Purkinje cell complex spike synchronicity in the rat cerebellum. J. Physiol. 470, 243-271.

Sugihara, I., Lang, E. J., and Llinás, R. (1995). Serotonin modulation of inferior olivary oscillations and synchronicity: a multiple-electrode study in the rat cerebellum. Eur. J. Neurosci. 7, 521-534.

Sugihara, I., and Shinoda, Y. (2004). Molecular, topographic, and functional organization of the cerebellar cortex: a study with combined aldolase $\mathrm{C}$ and olivocerebellar labeling. $J$. Neurosci. 24, 8771-8785.

Sugihara, I., Wu, H., and Shinoda, Y. (1999). Morphology of single olivocerebellar axons labeled with biotinylated dextran amine in the rat. J. Comp. Neurol. 414, 131-148.
Sugihara, I., Wu, H. S., and Shinoda, Y (2001). The entire trajectories of single olivocerebellar axons in the cerebellar cortex and their contribution to cerebellar compartmentalization. J. Neurosci. 21, 7715-7723.

Telgkamp, P., and Raman, I. M. (2002). Depression of inhibitory synaptic transmission between Purkinje cells and neurons of the cerebellar nuclei. J. Neurosci. 22, 8447-8457.

Teune, T.M., van der Burg, J., De Zeeuw, C. I., Voogd, J., and Ruigrok, T. J. (1998). Single Purkinje cell can innervate multiple classes of projection neurons in the cerebellar nuclei of the rat: a light microscopic and ultrastructural tripletracer study in the rat. J. Comp. Neurol. 392, 164-178.

Teune, T. M., van der Burg, J., van der Moer, J., Voogd, J., and Ruigrok, T. J. (2000). Topography of cerebellar nuclear projections to the brain stem in the rat. Prog. Brain Res. 124, 141-172.

Thach,W.T. (1968). Discharge of Purkinje and cerebellar nuclear neurons during rapidly alternating arm movements in the monkey. J. Neurophysiol. 31, 785-797.

Thach, W. T. (1975). Timing of activity in cerebellar dentate nucleus and cerebral motor cortex during prompt volitional movement. Brain Res. 88, 233-241.

Tolbert, D. L., Massopust, L. C., Murphy, M. G., and Young, P. A. (1976). The anatomical organization of the cerebello-olivary projection in the cat. J. Comp. Neurol. 170, 525-544.

Uusisaari, M., Obata, K., and Knopfel, T. (2007). Morphological and electrophysiological properties of GABAergic and non-GABAergic cells in the deep cerebellar nuclei. J. Neurophysiol. 97, 901-911.

Van der Want, J. J., Wiklund, L., Guegan, M., Ruigrok, T., and Voogd, J. (1989). Anterograde tracing of the rat olivocerebellar system with Phaseolus vulgaris leucoagglutinin (PHA-L).
Demonstration of climbing fiber collateral innervation of the cerebellar nuclei. J. Comp. Neurol. 288, 1-18.

Wassef, M., Simons, J., Tappaz, M. L., and Sotelo, C. (1986). Non-Purkinje cell GABAergic innervation of the deep cerebellar nuclei: a quantitative immunocytochemical study in C57BL and in Purkinje cell degeneration mutant mice. Brain Res. 399, 125-135.

Wetmore, D. Z., Mukamel, E. A., and Schnitzer, M. J. (2008). Lock-and-key mechanisms of cerebellar memory recall based on rebound currents. J. Neurophysiol. 100, 2328-2347.

Wu, H. S., Sugihara, I., and Shinoda, Y. (1999). Projection patterns of single mossy fibers originating from the lateral reticular nucleus in the rat cerebellar cortex and nuclei. J. Comp. Neurol. 411, 97-118.

Yartsev, M. M., Givon-Mayo, R., Maller, M., and Donchin, O. (2009). Pausing Purkinje cells in the cerebellum of the awake cat. Front. Syst. Neurosci. 3, 2. doi:10.3389/neuro.06.002.2009

Conflict of Interest Statement: The authors declare that the research was conducted in the absence of any commercial or financial relationships that could be construed as a potential conflict of interest.

Received: 23 June 2009; paper pending published: 13 July 2009; accepted: 08 November 2009; published online: 20 November 2009.

Citation: Baumel Y, Jacobson GA and Cohen D (2009) Implications of functional anatomy on information processing in the deep cerebellar nuclei. Front. Cell. Neurosci. 3:14. doi: 10.3389/neuro.03.014.2009 Copyright (C) 2009 Baumel, Jacobson and Cohen. This is an open-access article subject to an exclusive license agreement between the authors and the Frontiers Research Foundation, which permits unrestricted use, distribution, and reproduction in any medium, provided the original authors and source are credited. 